\title{
Estudio transversal de uso de servicios de salud mental en cinco ciudades del Perú
}

\author{
Marina Piazza, ${ }^{1,2}$ Fabián Fiestas $^{3}$
}

Artículo original

\begin{abstract}
Background

Neuropsichiatric diseases constitute the first cause of burden of disease in Peru.

\section{Objective}

The aim of this study was to describe 12 month mental health services utilization, of the 18 to 65 years old urban population in Peru.

\section{Method}

As part of the World Mental Health Survey Initiative (WMHSI), this research used a multistage, clustered-area probability sample of 3930 18-65 years old household residents in Lima, Arequipa, Huancayo, lquitos and Tacna between July 2004 and December of 2005. The Instrument used was the Composite International Diagnostic Interview (CIDI) that produces DSM-IV/ICD-9 diagnoses by means of computerized algorithms.
\end{abstract}

\section{Results}

In the last 12 months, only one of every 5 people who had a mental disorder during this period received any treatment. The average number of treatment visits per year was only three. $9.7 \%$ of those with any mental disorder received appropriate treatment. People with low education had less access to minimally adequate treatment $(O R=0.1$, $95 \% \mathrm{Cl}=0.0,0.5)$. The group with substance use disorders had lower access to treatment in the last 12 months $(12.7 \%)$.

\section{Discussion and conclusion}

Peru presents a wide gap in mental health treatment. Mental Health treatment gap in Peru emphasizes the need to direct resources towards screening and treatment of mental disorders.

Key words: Prevalence, services, mental health, Peru.

\section{RESUMEN}

\section{Antecedentes}

La primera causa de carga de enfermedad en el Perú son las enfermedades neuropsiquiátricas.

\section{Objetivo}

El objetivo del presente estudio fue generar información acerca del uso de 12 meses de servicios de salud mental en la población adulta urbana de Perú.

\section{Método}

El Estudio Mundial de Salud Mental(EMSM)se desarrolló con una muestra probabilística y multietápica de 3930 participantes de 18 a 65 años de edad en Lima, Arequipa, Huancayo, lquitos y Tacna entre julio del 2004 y diciembre del 2005. Utilizó la Entrevista Diagnóstica Internacional Compuesta, que proporciona diagnósticos de acuerdo con el DSM-IV y el CIE-10.

\section{Resultados}

Sólo una de cada cinco personas que presentaron algún trastorno durante los últimos 12 meses recibió tratamiento durante ese período. En promedio, durante este tiempo recibieron tres atenciones. Sólo $9.7 \%$ de las personas con cualquier trastorno durante los últimos 12 meses recibió un tratamiento adecuado. Las personas con bajo nivel de educación tuvieron menor acceso a tratamiento mínimamente adecuado $(\mathrm{OR}=0.1 ; 95 \% \mathrm{Cl}=0.0,0.5)$. El grupo con trastornos de uso de sustancias presentó menor acceso a tratamiento en los últimos 12 meses (12.7\%).

\section{Discusión y conclusión}

En el Perú existe una brecha de atención de salud mental. Las personas de menor educación y las personas con trastorno de abuso de sustancias son el grupo de menor acceso a cualquier tipo de tratamiento adecuado. La brecha de atención pone en evidencia la urgente necesidad de dirigir atención y recursos hacia la detección y tratamiento oportuno de las enfermedades mentales en el Perú.

Palabras clave: Prevalencia, servicios, trastornos mentales, Perú.

1 Centro Nacional de Salud Pública, Instituto Nacional de Salud. Lima, Perú.

2 Facultad de Salud Pública y Administración, Universidad Peruana Cayetano Heredia. Lima, Perú.

3 Instituto de Evaluación de Tecnologías Sanitarias (IETSI) del Seguro Social de Salud (ESSALUD). Lima, Perú.

Correspondencia: Marina Piazza. Av. Defensores del Morro 2268, Lima 9, Perú. Teléfono: 999 607-630. E-mail: mpiazza@ins.gob.pe; piazzamarina@gmail.com Recibido primera versión: 22 de agosto de 2014. Segunda versión: 14 de mayo de 2015. Tercera versión: 2 de julio de 2015 . Aceptado: 3 de agosto de 2015 


\section{INTRODUCCIÓN}

Los trastornos mentales son comunes en la población peruana. Según reportamos en un estudio previo, el 29\% ha presentado algún trastorno mental en su vida ${ }^{1}$ y $13.5 \%$ presentó al menos uno de estos trastornos en el último año. ${ }^{2}$ Durante este período, el grupo más frecuente es el de trastornos de ansiedad (fobia específica, fobia social y trastorno de ansiedad de separación), seguido por trastornos del estado de ánimo (depresión mayor, distimia, trastorno bipolar), control de impulsos (trastorno explosivo intermitente, trastorno por déficit de atención e hiperactividad, trastorno oposicionista desafiante) y uso de sustancias (trastorno por uso de alcohol u otras sustancias). ${ }^{2}$

La alta prevalencia y su cronicidad explican que los trastornos neuropsiquiátricos representen la primera causa de carga de enfermedad en el Perú, lo que genera 16\% del total de años perdidos de vida saludable ajustados por discapacidad. ${ }^{3}$ Sin embargo, sólo el $2 \%$ del presupuesto público del sector salud se designa al cuidado y atención de la salud mental. ${ }^{4}$ Asimismo, se ha reportado que los trastornos por uso de alcohol generan al país pérdidas económicas de aproximadamente 254 millones de dólares al año. ${ }^{5}$

No obstante, los recursos humanos con capacidad para atender este tipo de problemas son escasos. Por cada 100000 habitantes, se cuenta sólo con dos psiquiatras, seis enfermeras, cuatro psicólogos y un trabajador social. Estos profesionales se concentran en Lima, donde ejerce el $82 \%$ de los psiquiatras del sistema público. ${ }^{6}$

La escasez de recursos humanos e infraestructura, limita el acceso a servicios de salud mental. Así, en Lima, del total de la población que reconoce haber padecido problemas de salud mental, sólo el $24.5 \%$ accedió a los servicios de salud. ${ }^{7}$ En el caso de las regiones de Sierra y Selva, fue significativamente menor al encontrado en Lima $(13.9 \%$ y $14.3 \%$ respectivamente). ${ }^{8,9}$

El objetivo de este estudio fue generar información acerca del uso de servicios de salud mental durante los últimos 12 meses en cinco ciudades del Perú.

\section{MÉTODO}

El presente estudio usa los datos de la Encuesta Mundial de Salud Mental en el Perú (EMSM-Perú), que utilizó una metodología ampliamente descrita en la bibliografía internacional. ${ }^{10}$ En Latinoamérica, además del Perú, han participado México, Brasil y Colombia.

\section{Diseño}

El ENSM-Perú tiene un diseño transversal, probabilístico, multietápico y estratificado. La cobertura geográfica fue el área urbana de las siguientes ciudades: Arequipa, Chiclayo,
Lima Metropolitana (todas de la costa peruana), Huancayo (sierra), Iquitos (selva). La unidad de investigación se formó con residentes habituales de 18 a 65 años de edad.

El marco muestral utilizado proviene del Precenso de 1999. ${ }^{11}$ El diseño muestral contempló estratificación de acuerdo con criterios geográficos y socioeconómicos. Mediante una tabla de equivalencia, se subdividió el marco muestral de cada ciudad en dos subestratos: el subestrato consolidado que incluía los conglomerados del marco con base en el censo de 1993, y el subestrato de crecimiento que incluía los conglomerados surgidos después del censo. La muestra fue diseñada para generar resultados estadísticamente confiables para los ámbitos geográficos del área metropolitana de Lima y Callao y las ciudades de Huancayo, Iquitos, Arequipa y Chiclayo. En cada ciudad, la unidad primaria de muestreo fue la sección censal urbana, conformada por una o más manzanas o partición de manzanas de gran magnitud de viviendas. En promedio, una UPM contenía 80 viviendas particulares. Se requirió un listado de viviendas dentro de la UPM (conglomerado) antes de seleccionar la muestra final de viviendas y la selección de la persona a encuestar.

La unidad secundaria de muestreo estuvo constituida por las viviendas particulares existentes en los conglomerados. Se realizó selección aleatoria de 10 unidades de viviendas del listado del segmento de área. Por último, la unidad terciaria de muestreo seleccionó personas de 18 a 65 años de edad, residentes habituales de los hogares. Se enumeró a las personas elegibles, residentes en el hogar, seleccionando a una persona al azar. No hubo sustitución si la persona seleccionada no pudo ser entrevistada. La asignación de la muestra por ciudad investigada se realizó con el método de la Asignación de Potencia en atención al número de viviendas de cada ámbito y la varianza de las principales características a investigar.

\section{Participantes}

La población investigada fue definida como el conjunto de todas las viviendas particulares y sus residentes habituales de 18 a 65 años de edad de los ámbitos considerados para el estudio. Se excluyó a las personas residentes en instituciones públicas o privadas, cuarteles, reservaciones militares o de cualquier otro tipo de forma de vida que no sea familiar. El instrumento fue dividido en dos partes: 3930 personas contestaron sólo la parte I; de éstos, 1801 respondieron la parte I y la parte II del instrumento. El presente estudio incluyó a las 1801 personas que completaron ambas partes del instrumento (figura 1).

\section{Variables}

El instrumento utilizado fue la Entrevista Diagnóstica Internacional Compuesta, versión certificada 15 CAPI. ${ }^{12} \mathrm{El}$ CIDI CAPI es una entrevista diagnóstica estructurada apli- 
cada en una entrevista cara a cara por encuestadores, con apoyo de una computadora portátil. Según los criterios del Manual Diagnóstico y Estadístico de los Trastornos Mentales-IV (DSM-IV) y de la Clasificación Internacional de Enfermedades (CIE-10), diagnósticos de alguna vez en la vida, durante los últimos 12 meses y durante los últimos 30 días de 23 diagnósticos. Otras áreas investigadas por el instrumento son uso de servicios, características sociodemográficas, empleo, finanzas, factores del matrimonio e hijos, redes sociales, carga familiar, condiciones médicas crónicas, farmacoepidemiología y discapacidad.

Para maximizar la información recogida y minimizar el tiempo de recolección de información, el instrumento estuvo organizado en Parte I y Parte II. Todos los sujetos respondieron la primera parte del instrumento. Sólo contestaron la segunda parte quienes presentaron en la sección de tamisaje síntomas de algún trastorno. Asimismo, se seleccionó para responder a la segunda parte del cuestionario en forma aleatoria al 25\% de quienes no presentaron síntomas en el tamisaje.

El total de personas que respondieron a ambas partes fue de 1801, que constituyeron la muestra del presente estudio.

Las propiedades psicométricas del CIDI han sido descritas en diversas publicaciones. ${ }^{13} \mathrm{El}$ instrumento ha demostrado tener adecuada validez y confiabilidad entre entrevistadores test-retest. Su traducción al español fue realizada siguiendo las guías desarrolladas por la OMS, basándose en materiales que ya habían sido traducidos (ICD-10, DSM-IV, SF-36, WHO-DAS), en pruebas cognitivas de la comprensión de los reactivos, en juicios de expertos y en retraducción de reactivos seleccionados y de los términos utilizados en las secciones clínicas. El EMSM-Perú desarrolló una prueba piloto para ajustar los instrumentos.

La recolección de datos fue realizada por el Instituto Nacional de Estadística e Informática entre los meses de julio del año 2004 y diciembre del año 2005. La tasa de respuesta ponderada fue de $90.2 \%$.

Para asegurar el control de calidad de la información, se implementó extensa supervisión de campo. Se contó con el apoyo del Instituto Nacional de Psiquiatría Ramón de la Fuente Muñiz, de México, para la obtención de indicadores de calidad de la información y de control-cobertura del entrevistador. Se emplearon programas estadísticos para detectar posibles errores e inconsistencias relacionadas con los tiempos de los eventos y respuestas faltantes, así como para introducir los valores correctos cuando era posible. Estos programas fueron diseñados por el equipo coordinador del proyecto internacional de la Universidad de Harvard, que colaboró estrechamente con los investigadores de Perú en el control de calidad y certificación de la base de datos.

El tipo de servicios considerados en la variable tratamiento fue bastante amplio. Se incluyeron servicios especializados de salud mental general (brindados por psiquiatras, especialista de salud mental como psicólogo o consejero, por trabajador social y uso de servicio de ayuda telefónica de salud mental), así como aquellos ofrecidos en servicios de medicina general (médicos generales, enfermeras y otros profesionales no mencionados con anterioridad). Igualmente, esta categoría tratamiento incluye servicios de ayuda fuera del sector salud y aquellos clasificados como de medicina complementaria/alternativa que considera, además de la medicina alternativa, la complementaria, la espiritual o religiosa (ofrecidos por religiosos, consejeros espirituales, curanderos, grupos de autoayuda y grupos de ayuda por Internet).

Se caracterizó el tratamiento recibido basándose en la definición de Tratamiento Adecuado y Tratamiento Mínimamente Adecuado, de acuerdo con guías usadas por el estudio mundial de salud mental. ${ }^{14-16}$ La variable Tratamiento Adecuado en el último año fue definida de dos maneras: como ocho o más visitas en cualquier sector, o como cuatro o más visitas y un mes con medicación. Tratamiento Mínimamente Adecuado fue definido como cuatro o más visitas en cualquier sector, o dos o más visitas y un mes con medicación.

El acceso a tratamiento se estimó para cualquier tipo de procedimiento, de acuerdo con el número de visitas recibidas, así como para las definiciones de tratamiento adecuado y de tratamiento mínimamente adecuado. Asimismo, se exploró la posible asociación entre uso de servicios, tipo de trastorno y características demográficas.

Las variables sociodemográficas incluidas fueron edad, sexo, educación, ingreso y estado civil. La edad se categorizó como 18-29, 30-44, 45-54 y z55 años de edad. Educación, como Baja/Promedio-Bajo, Promedio-Alto, Alto. Ingreso, por su parte, como Bajo, Promedio-Bajo, Promedio-Alto y Alto. La variable estado civil se dividió en Soltero, Separado/Viudo/Divorciado y Casado/Conviviente, (cuadro 1).

\section{Análisis estadístico}

Los datos fueron ponderados para ajustarlos a la probabilidad diferencial de selección y de no respuesta. Se examinó el patrón de utilización de servicios estimando la proporción de personas en tratamiento. Entre aquellos en tratamiento se estimó la media, mediana y el número de visitas, así como la probabilidad de acceder a tratamiento adecuado y mínimante adecuado. Con el modelo de regresión logística multivariada, se estimó la asociación (razón de momios) entre factores sociodemográficos y recibir cualquier tratamiento en los últimos 12 meses con intervalos de confianza al 95\%. Los errores habituales se corrigieron por medio del método de Linearización de Taylor, utilizando el paquete estadístico Sudaan versión 8.0.1 (http:/ / www.rti.org/sudaan, visitado por última vez, 05.07.2014). Se utilizó la prueba de Chi-cuadrada de Wald para estimar la significancia de la regresión logística multivariada.

Se estimó la asociación entre tipo de trastorno y recibir tratamiento en los últimos 12 meses mediante el modelo de regresión logística multivariado, controlado por los 


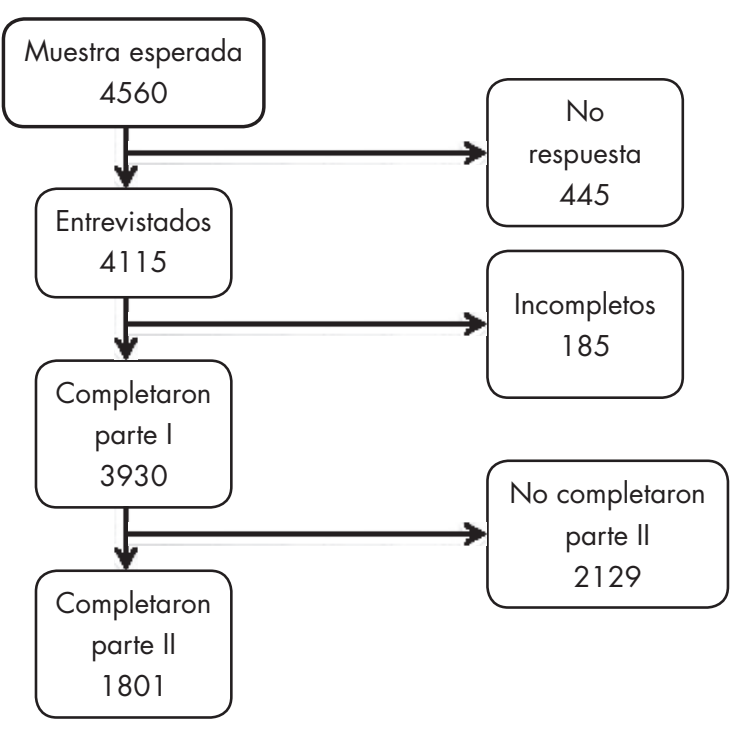

Figura 1. Flujograma de la muestra.

posibles confusores como edad, sexo, educación, ingreso y estado civil.

\section{Consideraciones éticas}

El EMSM-Perú fue aprobado por el Comité de Ética del Instituto Nacional de Salud del Perú. A los entrevistados se les aseguró que su participación era voluntaria y que podrían dejar de contestar si así lo desearan. También se les explicó que la información que proporcionaran sería confidencial y que sus datos personales no serían publicados. Al terminar la entrevista se entregó a los encuestados una lista de las instituciones de salud adonde podían acudir si deseaban hablar más de los temas tratados.

\section{RESULTADOS}

El tamaño de la muestra del EMSM-Perú fue de 3930 personas en la versión corta del instrumento y 1801 personas en su versión larga. En ambos casos, el 51.6\% fueron mujeres. El presente análisis incluyó a 360 personas que presentaron algún tipo de trastorno de salud mental durante los últimos 12 meses.

\section{Acceso a tratamiento para personas con trastornos de salud mental}

Sólo 19.1\% (error estándar, EE, 2.7) de las personas que presentaron algún trastorno durante los últimos 12 meses recibió algún tipo de atención durante ese período. El acceso al servicio varió de acuerdo al tipo de trastorno. Durante los últimos 12 meses solamente 27\% (error estándar, EE, 3.4) de aquellos que presentaron trastornos del estado de ánimo recibió atención (figura 2). La proporción que utilizó servicios

Cuadro 1. Correlatos demográficos de prevalencia de 12 meses de trastornos de salud mental DSM-IV y tratamiento

\begin{tabular}{|c|c|c|c|c|c|c|}
\hline \multirow[b]{2}{*}{ Variable } & \multicolumn{3}{|c|}{ Cualquier trastorno } & \multicolumn{3}{|c|}{ Cualquier tratamiento } \\
\hline & OR & IC $95 \%$ & & OR & IC 95\% & \\
\hline \multicolumn{7}{|l|}{ Sexo } \\
\hline Masculino & 1.00 & & & 1.00 & & \\
\hline Femenino & 1.27 & 0.96 & 1.69 & 2.06 & 1.41 & 3.00 \\
\hline \multicolumn{7}{|l|}{ Edad (años) } \\
\hline $18-29$ & 2.12 & 1.07 & 4.22 & 0.98 & 0.33 & 2.92 \\
\hline $30-44$ & 1.98 & 1.09 & 3.60 & 1.42 & 0.57 & 3.53 \\
\hline $45-54$ & 1.47 & 0.94 & 2.31 & 0.85 & 0.31 & 2.32 \\
\hline$\geq 55$ & 1.00 & & & 1.00 & & \\
\hline \multicolumn{7}{|l|}{ Ingreso } \\
\hline Bajo & 1.18 & 0.70 & 2.00 & 0.57 & 0.30 & 1.08 \\
\hline Promedio-bajo & 0.82 & 0.50 & 1.33 & 0.43 & 0.21 & 0.90 \\
\hline Promedio-alto & 0.89 & 0.50 & 1.57 & 0.45 & 0.25 & 0.80 \\
\hline Alto & 1.00 & & & 1.00 & & \\
\hline \multicolumn{7}{|l|}{ Estado civil } \\
\hline Casado/conviviente & 1.00 & & & 1.00 & & \\
\hline Separado/viudo/divorciado & 2.44 & 1.27 & 4.67 & 1.93 & 0.96 & 3.90 \\
\hline Soltero & 1.13 & 0.92 & 1.39 & 1.20 & 0.69 & 2.12 \\
\hline \multicolumn{7}{|l|}{ Educación } \\
\hline Baja & 1.13 & 0.74 & 1.73 & 0.77 & 0.29 & 2.01 \\
\hline Promedio-baja & 1.28 & 0.82 & 1.99 & 0.72 & 0.26 & 1.99 \\
\hline Promedio-alta & 1.09 & 0.73 & 1.64 & 0.80 & 0.42 & 1.50 \\
\hline Alta & 1.00 & & & 1.00 & & \\
\hline
\end{tabular}

Analizado con la muestra de versión larga $(n=1801)$.

* Severidad definida como severa/moderada en comparación con leve ( $n=391$, en esta columna). 
Cuadro 2. Porcentaje de personas con trastorno que recibe tratamiento adecuado y tratamiento mínimamente adecuado durante los últimos 12 meses

\begin{tabular}{lccc}
\hline $\begin{array}{l}\text { Tipo de trastorno de salud mental presentado } \\
\text { durante los últimos } 12 \text { meses }\end{array}$ & $\begin{array}{c}\text { \% que recibe tratamiento } \\
\text { adecuado }\end{array}$ & $\begin{array}{c}\text { \% que recibe tratamiento } \\
\text { mínimamente adecuado }\end{array}$ & $\begin{array}{c}\text { Personas con trastornos } \\
n\end{array}$ \\
\hline Cualquier trastorno de ansiedad*** & 5.8 (EE 3.5) & 47.8 (EE 6.6) & 53 \\
Cualquier trastorno estado de ánimo** & 9.9 (EE 5.1) & 57.5 (EE 7.6) & 39 \\
Cualquier trastorno*** & 9.7 (EE 2.6) & 50.3 (EE 5.2) & 78 \\
\hline
\end{tabular}

* $\mathrm{EE}=$ Errores estándar.

** peso de parte l.

$\star * \star$ peso de parte II.

fue menor para personas con trastornos de ansiedad $(18.8 \%$, EE, 3.5). Las personas con trastornos de uso de sustancias fue el grupo con menor acceso al servicio de salud mental: sólo el $12.7 \%$ (EE, 5.2) de personas en este grupo recibió algún tipo de tratamiento en los 12 meses previos a la entrevista.

\section{Número de atenciones de salud mental recibidas}

El número de visitas que recibieron las personas con cualquier trastorno de salud mental durante los últimos 12 meses fue en promedio de sólo tres. El número de visitas recibidas fue similar para las personas con trastornos del estado de ánimo y personas con trastornos de ansiedad. El número de personas con trastornos de consumo de sustancias fue reducido $(n=57)$ y no permitió incluir este tipo de trastorno en el presente análisis.

\section{Tratamiento de salud mental adecuado y mínimamente adecuado}

De acuerdo con esta definición, sólo 9.7\% de las personas con cualquier trastorno durante los últimos 12 meses recibió tratamiento adecuado (cuadro 2). Esta proporción fue mayor para personas con cualquier trastorno del estado de ánimo.

Cuadro 3. Características demográficas asociadas a recibir tratamiento durante los últimos 12 meses

\begin{tabular}{|c|c|c|}
\hline & \multicolumn{2}{|c|}{ Porcentaje de personas con trastorno que recibió tratamiento } \\
\hline & $\begin{array}{c}\text { Adecuado } \\
\text { en los últimos } 12 \text { meses }\end{array}$ & $\begin{array}{l}\text { Mínimamente adecuado } \\
\text { en los últimos } 12 \text { meses }\end{array}$ \\
\hline \multicolumn{3}{|l|}{ Edad } \\
\hline $18-29$ & $0.7(0.2,2.2)$ & $0.3(0.1,1.6)$ \\
\hline $30-44$ & $0.7(0.2,2.9)$ & $0.3(0.1,1.6)$ \\
\hline $45-54$ & $0.4(0.1,2.1)$ & $3.4(0.2,55.1)$ \\
\hline $55+$ & $1.0(1.0,1.0)$ & $1.0(1.0,1.0)$ \\
\hline Significancia de prueba Wald-Chi & $3 \mathrm{gl}^{*}=1.4, \mathrm{p}=0.712$ & $3 \mathrm{gl}=18.0, p<0.001$ \\
\hline \multicolumn{3}{|l|}{ Educación } \\
\hline Baja/promedio-bajo & & $0.1(0.0,0.5)$ \\
\hline Promedio-alto & & $1.2(0.3,5.5)$ \\
\hline Alto & & $1.0(1.0,1.0)$ \\
\hline Significancia de prueba Wald-Chi & & $2 \mathrm{gl}=19.3, p<0.001$ \\
\hline \multicolumn{3}{|l|}{ Ingreso } \\
\hline Bajo & $0.4(0.2,0.8)$ & $1.0(0.2,6.1)$ \\
\hline Promedio-bajo & $0.5(0.2,1.3)$ & $1.1(0.1,9.9)$ \\
\hline Promedio-alto & $0.5(0.1,1.5)$ & $0.7(0.1,5.8)$ \\
\hline Alto & $1.0(1.0,1.0)$ & $1.0(1.0,1.0)$ \\
\hline Significancia de prueba Wald-Chi & $3 \mathrm{gl}=6.9, \mathrm{p}=0.076$ & $3 \mathrm{gl}=0.3, p=0.950$ \\
\hline \multicolumn{3}{|l|}{ Estado civil } \\
\hline Soltero & $1.1(0.4,2.9)$ & $0.6(0.3,1.5)$ \\
\hline Separado/viudo/divorciado & $1.1(0.4,3.1)$ & $1.1(0.3,4.1)$ \\
\hline Casado/conviviente & $1.0(1.0,1.0)$ & $1.0(1.0,1.0)$ \\
\hline Significancia de prueba Wald-Chi & $2 \mathrm{gl}=0.1, \mathrm{p}=0.957$ & $2 \mathrm{gl}=1.3, p=0.509$ \\
\hline \multicolumn{3}{|l|}{ Sexo } \\
\hline Masculino & $0.8(0.4,1.9)$ & $0.9(0.4,1.9)$ \\
\hline Femenino & $1.0(1.0,1.0)$ & $1.0(1.0,1.0)$ \\
\hline Significancia de prueba Wald-Chi & $1 \mathrm{gl}=0.2, \mathrm{p}=0.638$ & $1 \mathrm{gl}=0.1, p=0.759$ \\
\hline
\end{tabular}

* $\mathrm{gl}=$ grados de libertad. 
Cuadro 4. Tipo de Trastorno Asociado a Recibir Tratamiento durante los últimos 12 meses

\begin{tabular}{lcc}
\hline & \multicolumn{2}{c}{ Probabilidad de recibir tratamiento } \\
\cline { 2 - 3 } & $\begin{array}{c}\text { Adecuado entre personas } \\
\text { con trastorno en los últimos } 12 \text { meses }\end{array}$ & $\begin{array}{c}\text { Mínimamente adecuado entre personas } \\
\text { con trastorno en los últimos } 12 \text { meses }\end{array}$ \\
\hline Cualquier trastorno de ansiedad & $1.3(0.4,4.0)$ & $0.5(0.1,2.5)$ \\
Sí & $1.0(1.0,1.0)$ & $1.0(1.0,1.0)$ \\
No & $1 \mathrm{gl}=0.2, \mathrm{p}=0.663$ & $1 \mathrm{gl}=0.9, \mathrm{p}=0.339$ \\
Significancia de prueba Wald-Chi & $\mathbf{2 . 6 ( 1 . 3 , 5 . 1 )}$ & $0.7(0.1,5.6)$ \\
Cualquier trastorno del estado de ánimo & $1.0(1.0,1.0)$ & $1.0(1.0,1.0)$ \\
Sí & $1 \mathrm{gl}=8.0, \mathrm{p}=0.005$ & $1 \mathrm{gl}=0.2, \mathrm{p}=0.680$ \\
No & & $1.4(0.2,12.0)$ \\
Significancia de prueba Wald-Chi & $0.8(0.2,2.8)$ & $1.0(1.0,1.0)$ \\
Cualquier trastorno por uso de sustancias & $1.0(1.0,1.0)$ & $1 \mathrm{gl}=0.1, \mathrm{p}=0.741$ \\
Sí & $1 \mathrm{gl}=0.1, \mathrm{p}=0.732$ & \\
No & & \\
Significancia de prueba Wald-Chi & & \\
\hline
\end{tabular}

${ }^{*} \mathrm{gl}=$ grados de libertad.

Para la definición de Tratamiento Mínimamente Adecuado, se observó que el acceso durante los últimos 12 meses fue de $50.2 \%$ para personas que presentaron cualquier trastorno de salud mental durante ese período. Comparando la proporción que accedió a Tratamiento Mínimamente Adecuado por tipo de trastorno, se evidencia que éste fue de $57.4 \%$ para personas con cualquier trastorno del estado de ánimo y de $47.7 \%$ para aquellas con cualquier trastorno de ansiedad. Nuevamente, el escaso tamaño muestral de personas con trastornos de consumo de sustancias $(n=57)$ impidió hacer inferencias estadísticas para este grupo.

\section{Características demográficas asociadas a recibir tratamiento de salud mental durante los últimos 12 meses}

Las personas con bajo nivel de educación tuvieron menor acceso a Tratamiento Mínimamente Adecuado $(\mathrm{OR}=0.1$; $95 \% \mathrm{CI}=0.0,0.5)$ en comparación con aquellos con un nivel alto de educación. No se encontró asociación entre el acceso a tratamiento durante los últimos 12 meses y otras características demográficas analizadas, como edad, ingreso, estado civil y sexo (cuadro 3).

\section{Tipo de trastorno asociada a recibir tratamiento de salud mental durante los últimos 12 meses}

Se evaluó si entre las personas con trastornos de salud mental durante los últimos 12 meses existían diferencias por tipo de trastorno respecto al acceso a tratamiento. Las personas que presentaron cualquier trastorno del estado de ánimo durante los últimos 12 meses tuvieron $2.6 \%$ de mayor probabilidad de acceder a tratamiento adecuado que quienes presentaron un diferente tipo de trastorno, tal como se presenta en el cuadro 4. Por otra parte, la probabilidad de acceso a tratamiento fue similar entre quienes presentaban cualquier trastorno de ansiedad y quienes no presentaban este tipo de trastorno (i.e., $\mathrm{p} \geq 0.05$ ). Igualmente, la probabilidad de acceder a tratamiento fue similar entre quienes presentaban algún trastorno de consumo de sustancias en comparación con quienes no lo presentaban (i.e., $\mathrm{p} \geq 0.05$ ) (cuadro 4, figura 2).

\section{DISCUSIÓN Y CONCLUSIÓN}

El presente estudio puso en evidencia que la mayor parte de las personas con un trastorno de salud mental presente durante los últimos meses no reciben ningún tipo de ayuda. Es decir, del total de personas con trastornos mentales, sólo 19\% accedieron a servicios de tratamiento en el último año. Se observó que el uso de servicios varía de manera importante de acuerdo con el tipo de trastorno que se presenta. El grupo de trastorno con mayor acceso es el de trastornos de ansiedad, en el cual, por cada tres personas con el trastorno, solamen-

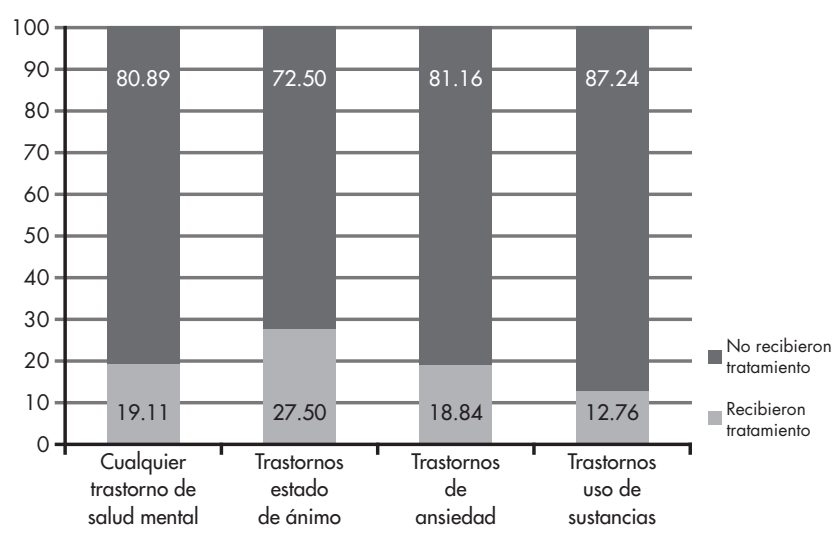

Figura 2. Porcentajes de personas con enfermedad mental que recibió tratamiento durante los últimos 12 meses. 
te una recibe tratamiento. El grupo de trastornos de menor acceso son los de uso de sustancias, donde sólo una de cada 10 personas recibe algún tipo de ayuda en los últimos doce meses. Estas cifras evidencian que, en general, el acceso a servicios de tratamiento de personas con trastornos mentales es muy limitado aun para la población urbana en el Perú. Además, se evidencia que hay diferencias por tipo de trastorno mental, puesto que la menor oportunidad de recibir ayuda es para las personas con trastornos de consumo de sustancias.

Otros estudios epidemiológicos de salud mental en el Perú han reportado que, entre residentes urbanos que manifiestan necesidad de atención en salud mental durante los últimos seis meses, sólo entre el 15\% al 25\% recibió algún tipo de tratamiento. ${ }^{7-9}$ Dada la naturaleza crónica de los trastornos de salud mental, esta falta de atención representa una importante pérdida de oportunidad de modificar el curso de la enfermedad, traduciéndose en una innecesaria y evitable carga, así como en un considerable costo económico y social para el país.

El estudio diferenció el uso de los servicios de acuerdo con dos definiciones de tratamiento de guías clínicas basadas en evidencia: tratamiento adecuado y tratamiento mínimamente adecuado. De acuerdo con la definición de tratamiento adecuado, el estudio generó evidencia que sostiene que en las ciudades investigadas, por cada diez personas con trastornos de salud mental presentes durante los últimos 12 meses, solamente una de cada diez recibió algún tipo de tratamiento. Utilizando la definición de Tratamiento Mínimamente Adecuado, se observa que por cada 100 personas con necesidad de recibir tratamiento, sólo 50 lo reciben. Estos resultados colocan al Perú en una situación de inadecuación respecto a la provisión y uso de servicios en esta área prioritaria de salud. . $^{178}$

El segundo objetivo fue determinar los predictores del tratamiento de salud mental en el Perú. El estudio puso en evidencia que la oportunidad de acceder al tratamiento mínimamente adecuado es menor para las personas de menor nivel de educación. Estos resultados son consistentes con otros estudios epidemiológicos en el país, que refieren que la falta de dinero representó el principal motivo para no atenderse. En este caso, entre aquellos que refieren percibir la necesidad de recibir atención de salud mental, $47 \%$ expresó, como motivo principal de no recibirla, la falta de dinero. ${ }^{7-9}$ De igual manera, estos resultados son consistentes con los obtenidos por el Estudio de Salud Mental en México y en los Estudio en Estados Unidos. ${ }^{18,19}$ De esta manera, podemos observar que, ante la limitada oferta de servicios de salud mental desde instituciones públicas, la posibilidad de recibir alguna ayuda depende de la capacidad privada de financiamiento. Así, las personas con problemas de salud mental con menos recursos económicos se encuentran en una situación de desventaja, que incluso puede derivar en un gasto catastrófico por causa de una enfermedad mental. Esta situación contribuye a relegar y excluir aún más a esta población. Recientemente, el
Seguro Social de Salud, EsSalud, ha incorporado el tamisaje y tratamiento de algunos trastornos mentales dentro de su cobertura en todos los niveles de atención. ${ }^{20}$ Esta medida representa un avance importante para el $22 \%$ de la población peruana que cuenta con EsSalud. ${ }^{21}$

El presente estudio pone en evidencia que el acceso a los servicios adecuados y mínimantente adecuados es igualmente reducido para hombres que para mujeres. En contraste, el acceso a cualquier forma de tratamiento de salud mental fue mayor en mujeres que en hombres, si utilizamos la amplia definición presentada en la sección de métodos. Es decir, dada la limitada oferta de tratamiento para la enfermedad mental, las mujeres recurren a otras formas de ayuda en mayor medida que los hombres. Esta carencia de servicios formales puede llevar a la población a exponerse a métodos de tratamiento informales que pueden involucrar prácticas controversiales, algunas de las cuales llegan incluso a violar los derechos humanos.

Los sistemas de salud son exitosos cuando las personas se mantienen sanas. $\mathrm{O}$ bien, si se enferman y tienen acceso a los servicios sanitarios. En este caso, siguen su tratamiento, limitando el daño o rehabilitándose, y están satisfechas con los servicios que mejoran su calidad de vida y su nivel de salud. ${ }^{22} \mathrm{El}$ acceso a servicios de salud mental de calidad representa un derecho respaldado por el Pacto Internacional de Derechos Económicos, Sociales y Culturales suscrito por el Perú en el año $1978 .{ }^{23}$ Con esta convención, el estado reconoce el derecho de cada peruano de alcanzar el estado más alto de salud física y mental y de proveer las medidas necesarias para alcanzar este objetivo. De igual manera, la Constitución Política del Perú, en su artículo 7, establece el derecho a la protección de la salud de las personas con discapacidad física y mental. Asimismo, el país cuenta con los Lineamientos de Acción en Salud Mental y la Estrategia Sanitaria Nacional de Salud Mental y Cultura de Paz, lo que significa un importante marco normativo de acción. Sin embargo, el presente estudio resalta la brecha en la traducción de este marco normativo a la provisión de servicios.*

Aunque el presente estudio no ha abarcado características del tratamiento recibido respecto al uso de medicamentos, es importante señalar que se ha descrito que alrededor del $50 \%$ de los establecimientos de salud no tienen disponible antidepresivos ni antipsicóticos, y que 90\% o más no cuentan con otras cuatro clases de medicamentos psiquiátri$\cos ^{24}$ Es decir, que la disponibilidad de medicamentos utilizados en el tratamiento de la enfermedad mental en el Perú es inferior a la de otros países de bajos o medianos ingresos.

Una estrategia para disminuir la brecha de atención en salud mental es la de integrar estos servicios con aquellos basados en atención primaria, con personal de salud no especia-

\footnotetext{
* Ministerio de Salud del Perú. Estrategia sanitaria nacional de salud mental y cultura de paz. Lima: Ministerio de salud-Dirección General de Promoción de la salud, 2006
} 
lizado que realiza el tamizaje y diagnóstico, y derivar oportunamente al paciente para que cuente con el asesoramiento de un especialista de salud mental que monitoree el tratamiento y maneje los casos más complejos. ${ }^{25}$ Desde esta perspectiva, el Ministerio de Salud del Perú (MINSA) inició en el 2008 la generación de guías de práctica clínica para depresión, psicosis, violencia basada en género y problemas relacionados con el uso de sustancias psicoactivas, desactualizadas, y poco usadas por el personal de salud. ${ }^{26}$ El desafío no sólo es fortalecer la oferta y la calidad de los servicios, sino transformar los sistemas de salud implementando aproximaciones basadas en la evidencia para el cuidado integrado y eficiente de la enfermedad mental y otras condiciones crónicas.

Una limitación del presente informe está dada por los datos recogidos en el 2004 y 2005. Por otro lado, la muestra solamente incluyó a la población urbana donde la brecha de acceso debe ser mayor que en el contexto urbano.

En conclusión, en el Perú existe una brecha de atención de salud mental que se traduce en un bajo porcentaje de la población urbana que accede a tratamiento mínimamente adecuado, y a un menor porcentaje que accede a un tratamiento adecuado. Las personas de menor educación son un grupo de mayor vulnerabilidad respecto a su acceso a un tratamiento de salud. Las personas con trastorno de abuso de sustancias son el grupo de menor acceso a cualquier tipo de tratamiento adecuado.

\section{Financiamiento}

La Encuesta Mundial de Salud Mental en Perú se desarrolla en colaboración con la Encuesta Mundial de Salud Mental (WMH) de la Organización Mundial de la Salud, la cual contó con financiamiento del Instituto Nacional de Salud Mental de los Estados Unidos (NIMH; R01 MH070884), la Fundación John D. y Catherine T. MacArthur, la Fundación Pfizer, el Servicio de Salud Pública de Estados Unidos (R13-MH066849, R01-MH069864, y R01 DA016558), el Centro Internacional Fogarty (FIRCA R03-TW006481), la Organización Panamericana de la Salud, y las compañías farmacéuticas Eli Lilly, Ortho-McNeil, GlaxoSmithKline y Bristol-Myers Squibb. Agradecemos al personal de los Centros de Coordinación de Recojo y Análisis de datos del WMH el apoyo en la implementación del trabajo de campo y consultoría en el análisis de datos. Ninguno de los fundadores participó en el diseño, análisis, interpretación o preparación de este artículo. La lista completa de publicaciones de WMH se encuentra en el enlace http:/ / www.hcp.med.harvard.edu/wmh. El Estudio Mundial de Salud Mental en Perú fue financiado por el Instituto Nacional de Salud (INS) del Ministerio de Salud del Perú (MINSA), con apoyo adicional de la Organización Panamericana de la Salud.

\section{Conflicto de intereses}

Los autores declararon no tener conflicto de intereses.

\section{Agradecimientos}

Agradecemos a Ron Kessler, Nancy Sampson y Colleen Bouzan (Universidad de Harvard), María Elena Medina-Mora y Guilherme Borges (Instituto Nacional de Psiquiatría), Jorge Rodríguez y
María Edith Baca (Organización Panamericana de la Salud), Sergio Aguilar Gaxiola (Universidad Estatal de California en Fresno) y al equipo técnico local que participó en la revisión del instrumento durante la fase inicial del estudio.

\section{REFERENCIAS}

1. Fiestas F, Piazza M. Prevalencia de vida y edad de inicio de trastornos psiquiátricos en el Perú urbano: estudio epidemiológico en cinco ciudades de la costa, sierra y selva del Perú. Revista Peruana de Medicina Experimental y Salud Pública Rev Perú Med Exp Salud Pública 2014; 31(1):39-47.

2. Piazza M, Fiestas F. Prevalencia de trastornos mentales en el último año y uso de servicios: Resultados del Estudio Mundial de Salud Mental en El Perú. Rev Perú Med Exp Salud Pública 2014; 31(1):30-38.

3. Velásquez A. La carga de enfermedad y lesiones en el Perú y las otras prioridades del plan esencial de aseguramiento universal. Rev Perú Med Exp Salud Pública 2009;26:222-231.

4. Ministerio de Salud. Informe sobre los servicios de salud mental del subsector Ministerio de Salud del Perú 2008. Lima: Ministerio de Salud-OPS-OMS; 2009.

5. DEVIDA, 2010: Impacto social y económico del consumo de drogas en el Perú.

6. Kohn Robert et al. Los trastornos mentales en América Latina y el Caribe: asunto prioritario para la salud pública. Rev Panam Salud Pública [online]. 2005, vol.18, n.4-5, pp. 229-240. ISSN 1020-4989. http:// dx.doi.org/10.1590/S1020-49892005000900002 (revisado el 5.8.2014).

7. Instituto Nacional de Salud Mental. Estudio epidemiológico en salud mental 2002. Anales Salud Mental 2002;18(1, 2):13-81.

8. Instituto Nacional de Salud Mental. Estudio epidemiológico de salud mental en la sierra peruana 2003. Anales Salud Mental 2003;19(1,2):25102.

9. Instituto Nacional de Salud Mental. Estudio epidemiológico de salud mental en la selva peruana 2004. Anales Salud Mental 2004;21(1,2):2598.

10. Kessler RC, Ustun TB. The World Mental Health (WMH) Survey Initiative Version of the World Health Organization (WHO) Composite International Diagnostic Interview (CIDI). Int J Methods Psychiatr Res 2004;13(2):93-121.

11. Instituto Nacional de Estadística e Informática. Censos Nacionales 2007: XI de Población y VI de Vivienda. Lima 2008.

12. Andrew G, Peters L. The psychometric properties of the Composite International Diagnostic Interview. Soc Psychiatry Psychiatr Epidemiol (1998)33:80-8.

13. World Health Organization. Procedures for the development of new language versions of the WHO Composite International Diagnostic Interview (WHO-CIDI). Ginebra; 1998.

14. American Psychiatric Association. Practice guideline for treatment of patients with schizophrenia. 2nd ed. Washington, DC: American Psychiatric Association Press; 2004.

15. American Psychiatric Association. Practice guideline for treatment of patients with bipolar disorder. 2nd ed. Washington, DC: American Psychiatric Association Press; 2002.

16. American Psychiatric Association. Practice guideline for treatment of patients with panic disorder. Washington, DC: American Psychiatric Association Press; 1998.

17. Organización Panamericana de la Salud. Epidemiología de los trastornos mentales en América Latina y el Caribe. Washington, D.C.: OPS; 2009.

18. Medina-Mora ME, Borges G, Benjet C, Lara C et al. Prevalencia de trastornos mentales y uso de servicios: Resultados de la Encuesta Nacional de Epidemiología Psiquiátrica en México. Salud Mental 2003;26(4):1-16. 
19. Wang PS, Lane M, Kessler RC, Olfson M et al. Twelve-month use of mental health services in the U.S.: Results from the National Comorbidity Survey Replication (NCS-R). Arch Gen Psychiatry 2005;62(6)629640.

20. Seguro Social de Salud (EsSalud). Resolución de Gerencia General 1207- 2013: Cartera de servicios de salud de complejidad creciente del Seguro Social de Salud (EsSalud). Lima: Gerencia Central de Prestaciones de Salud, EsSalud; 2013.

21. Instituto Nacional de Estadística e Informática. Condiciones de vida en el Perú: julio-agosto-setiembre Lima, Perú; 2011.

22. Subsanar las desigualdades de una generación: alcanzar la equidad sanitaria actuando sobre los determinantes sociales de la salud. Informe final de la Comisión sobre Determinantes Sociales de la Salud. Ginebra: OMS; 2008.

23. Pacto Internacional de Derechos Económicos, Sociales y Culturales adoptado y abierto a la firma, ratificación y adhesión por la Asamblea
General en su resolución 2200 A (XXI), de 19 de diciembre de 1966. Aprobado por Perú por Decreto Ley N ${ }^{\circ} 22129$ de 28 de marzo de 1978. Instrumento de adhesión de 12 de abril de 1978. Depositado el 28 de abril de 1978. Fecha de entrada en vigencia el 28 de julio de 1978.

24. Hodgkin D, Piazza M, Crisante M, Gallo C et al. Disponibilidad de medicamentos psicotrópicos en establecimientos del Ministerio de Salud del Perú. Rev Perú Med Exp Salud Pública 2014;31(4):660-668.

25. World Health Organization and Calouste Gulbenkian Foundation. Integrating the response to mental disorders and other chronic diseases in health care systems. World Health Organization. Ginebra: 2014.

26. Gálvez-Buccollini JA, Fiestas F. Necesidad de evaluar las guías clínicas peruanas de tratamiento para trastornos mentales. Rev Perú Med Exp Salud Pública 2011;28:698-699. 